\title{
Risk factors and Compliance of surviving sepsis campaign: A retrospective cohort study at tertiary care hospital
}

\author{
Farheen Yousuf ${ }^{1}$, Ayesha Malik2, \\ Ayesha Saba ${ }^{3}$, Sana Sheikh ${ }^{4}$
}

\begin{abstract}
Background and Objective: Sepsis is one of the leading causes of direct maternal mortality in Pakistan. It is recommended that the first three hours after the presentation are crucial. During this time implementation of surviving sepsis campaign resuscitation bundles reduces maternal mortality. Our objective was to assess the factors contributing to puerperal sepsis and the compliance of "surviving sepsis campaign resuscitation bundles in puerperal sepsis" for the management of puerperal sepsis.

Methods: This was a retrospective record review for five years from January 2011 -December 2015. All women who fulfilled the inclusion criteria of puerperal sepsis were included and data from their files were collected and entered in SPSS version 19.0. Mean and standard deviations were calculated for continuous variables while for categorical variable proportion and percentages were used.

Results: This retrospective record review in five years showed the 396 patients had P-sepsis, among them 44 patients had severe sepsis with organ dysfunction. The culture was positive in $26(59 \%)$ with trend of E-coli in $9(20 \%)$ Among them $12(27 \%)$ had serum lactate more than $\geq 4 \mathrm{mmol} / \mathrm{L}$. Central venous pressure monitoring with fluid resuscitation was done as per protocol of survival bundle given to all $12(100 \%)$, Vasopressin was needed in half of these patients $6(50 \%)$. Amid 44 patients of severe sepsis $29(66 \%)$ were admitted to special care, while $15(34 \%)$ required intensive care admissions. Our $7(16 \%)$ patients failed to survive. All of them had multi-organ failure.

Conclusion: There was moderate adherence of modified surviving sepsis campaign resuscitation bundles. Further improvement in compliance is warranted.
\end{abstract}

KEYWORDS: Sepsis, Surviving sepsis campaign, Puerperal pyrexia, Maternal mortality.

doi: https://doi.org/10.12669/pjms.38.1.3992

How to cite this:

Yousuf F, Malik A, Saba A, Sheikh S. Risk factors and Compliance of surviving sepsis campaign: A retrospective cohort study at tertiary care hospital. Pak J Med Sci. 2022;38(1):90-94. doi: https://doi.org/10.12669/pjms.38.1.3992

This is an Open Access article distributed under the terms of the Creative Commons Attribution License (http://creativecommons.org/licenses/by/3.0), which permits unrestricted use, distribution, and reproduction in any medium, provided the original work is properly cited.

1. Dr. Farheen Yousuf, FRCOG, MCPS(HPE), FCPS.

2. Dr. Ayesha Malik, MRCOG, FCPS.

3. Dr. Ayesha Saba, FCPS.

4. Dr. Sana Sheikh, Masters in Epidemiology and Biostatics

1-4: Department of Obstetrics and Gynecology,

Aga Khan University,

Karachi, Pakistan.

Correspondence:

Dr. Farheen Yousuf, FRCOG, MCPS(HPE), FCPS. Assistant Professor,

Department of Obstetrics and Gynecology, Aga Khan University, Karachi, Pakistan.

Postal Address: Apartment No. 205,

Premiere Residency, M.T. Khan Road, Karachi, Pakistan.

Email: Farheen.yousuf@aku.edu

* Received for Publication:

December 9, 2020

* $1^{\text {st }}$ Revision Received:

* $2^{\text {nd }}$ Revision Received:

* Final Revision Accepted:
March 16, 2021

July 9, 2021

July 29, 2021

\section{INTRODUCTION}

Millennium development goal (MDG) five was to reduce maternal mortality from background of 1990, by $75 \%$ by 2015 . There was much improvement by $50 \%$ reduction however, it was not up to the mark. ${ }^{1}$ The strategic development goal (SDG) targeted to reduce maternal mortality to $<70 / 100,000 .^{2}$ Pakistan reports showed the maternal mortality is $178 / 100,000$, this is far behind then the target. ${ }^{1}$ A study in 2014 reported as hemorrhage leads to $27 \%$ while sepsis causes $11 \%$ of maternal deaths. ${ }^{3}$ Similarly a systematic review also reported, sepsis as the direct maternal mortality in Asia by $11.6 \%$ while in developed countries it is only $2.1 \%{ }^{4}$ 
The exact burden of sepsis is difficult to gauge due to differences in definition. In 2019 definition of sepsis and septic shock has been revised at the third international consensus sepsis definition (Sepsis-3). ${ }^{5}$ There is a lot of literature which highlighted the risk factors associated with sepsis like un-booked, multiparity, anemia, caesarean section and so on and forth. ${ }^{6}$ However still we fail to achieve any improvement. Dellinger et al.'s study reported that puerperal pyrexia and sepsis are highly preventable problems occurring among the leading causes of maternal morbidity and mortality in both developing and developed countries. ${ }^{7}$ The issuance of international guidelines for management of severe sepsis and septic shock called as "Modified surviving sepsis campaign resuscitation bundles in puerperal sepsis" in $2008,{ }^{8}$ followed by Royal College of obstetricians and gynecologists in 2012, released early warning signs for the diagnosis criteria for ICU admission. It strongly recommends that first three hours after presentation are very crucial. During these hours certain tasks are predefined, this includes initiation of broad-spectrum antibiotics within one hour after obtaining blood and other relevant cultures, measuring serum lactate. In case of serum lactate being higher than $4 \mathrm{mmol} / 1$ or hypotension, crystalloid should be started with the rate of $30 \mathrm{ml} / \mathrm{kg}$ in order to achieve central venous pressure of $\geq 8 \mathrm{mmHg}$ and mean arterial pressure $\geq 65 \mathrm{~mm} \mathrm{Hg}$. Vasopressors should also be considered, if patient is not responding to fluid resuscitation. Central venous oxygen saturation should also be maintained at $\geq 70 \%, 9,10$

Pakistan has a high mortality due to puerperal sepsis. We are not sure that our patients with Puerperal sepsis are properly managed in light of international guidelines. There is no study published so far that focus on these lines of management in our region. Hence this study will assess the factors contributing to puerperal sepsis and compliance of "Modified surviving sepsis campaign resuscitation bundles in puerperal sepsis" for the management of puerperal sepsis. Furthermore, it also measures puerperal sepsis related maternal mortality and morbidity at Aga Khan university Hospital. The results of this study and recommendations in the light of this data will help in early diagnoses and treatment of cases with puerperal sepsis. We anticipate that this study will improve awareness in caregivers along with its implementation makes revolutionary improvement in maternal morbidity and mortality due to puerperal sepsis.

\section{METHODS}

This was a retrospective record review for five years from January 2011 to December 2015. All women who were admitted with suspicion of puerperal sepsis during pregnancy till 42 days after delivery were recruited and data from their files were collected. Women who were immunocompromised due to any other prior illness or using immunosuppressant were excluded. Puerperal sepsis is defined as the infection of the genital tract occurring at labor or within 42 days of the postpartum period. Among these patients who have had sepsis-induced organ dysfunction or tissue hypoperfusion were labelled as Severe sepsis. Septic shock is defined as the persistence of hypoperfusion despite adequate fluid replacement therapy along with constant severe abdominal pain and tenderness unrelieved by usual analgesia.

All patients who developed sepsis during pregnancy, labor or within 42 days after delivery were included in this study. Their files were reviewed. Details like age, gestational age, and mode of delivery, use of antibiotics, culture results, fluid therapy and serum lactate level were retrieved. Their course of hospital stays and management from the first encounter in emergency room were taken along with the details of time about what, when, who and how the management of P-sepsis was done, were recorded from the files

Data management and analysis: Data was entered and analyzed inStatistical PackageforSocialSciences (SPSS) version 19.0. Mean and standard deviations were calculated for continuous variables such as maternal age, gestational age at delivery. Clinical Presentations, culture results and compliance of sepsis surviving bundle were calculated in percentage. Permission from ethical review board of the institution was taken with reference number (3528-OBS-ERC-15). The information was taken from medical records remained confidential. Patient name and identity were not disclosed at any time.

\section{RESULTS}

There were 396 patients who presented to emergency with suspicion of puerperal sepsis. The mean age of patients with P-sepsis was 28.51(SD \pm 4.75$)$. The most prevalent contributing factor was anemia in 250(63\%) followed by diabetes in $77(19 \%)$ while preterm pre-labor rupture of membrane in $62(16 \%)$ of patients. Two third of patient delivered by Caesarean section. The common presentations were fever and 
Table-I: Clinical factors associated with P-sepsis.

\begin{tabular}{lc}
\hline & Total N 396(\%) \\
\hline Anemia & $250(63.1)$ \\
Diabetes & $77(19.4)$ \\
Vaginal discharge + Pelvic infection & $21(5.3)$ \\
PPROM & $62(15.7)$ \\
Mode of delivery & \\
Vaginal & $150(37.87)$ \\
Caesarean section & $246(62.12)$ \\
Fever & $257(64.9)$ \\
Rigors & $187(47.2)$ \\
Tachycardia & $254(64.1)$ \\
GIT symptoms & $34(8.58)$ \\
Lethargy & $103(26)$ \\
Breast abscess & $6(1.5)$ \\
UTI & $14(3.5)$ \\
RTI & $19(4.8)$ \\
Abdominal distention & $32(8.08)$ \\
Offensive vaginal discharge & $10(2.5)$ \\
Sub involution of uterus & $3(0.8)$ \\
Maternal Mortality due to & $7(15.90)^{*}$ \\
severe sepsis & \\
Sevel of Care & $29(65.90)^{*}$ \\
Intensive care unit & $15(34.09)^{*}$ \\
\hline
\end{tabular}

*N 44(Denominator).

tachycardia found in about $255(64 \%)$ of patients shown in Table-I.

Severe sepsis with organ dysfunction cases are shown in Table-II. There were $44(11 \%)$ of

Table-II: Sepsis associated with organ dysfunction and microorganism.

\begin{tabular}{ll}
\hline Organ dysfunction & $44(11.1)$ \\
Liver & $26(59.09)$ \\
Renal & $7(15.90)$ \\
Lungs & $2(4.54)$ \\
Multi organ failure & $9(20.45)$ \\
Organism Overall Culture positive & $26(59.09)$ \\
GAS & $5(11.36)$ \\
E-coli & $9(20.45)$ \\
Staphylococcus & $2(4.54)$ \\
Klebsella & $3(6.81)$ \\
Pseudomonas & $3(6.81)$ \\
MRSA & $1(2.27)$ \\
Acitenobacter & $3(6.81)$ \\
\hline
\end{tabular}

severe sepsis out of 396 patients. Further break up of organ failure revealed that liver was affected in $26(59 \%)$ followed by renal dysfuction $7(16 \%)$. Multi-organ involvement seen in $9(20 \%)$ of patients with severe sepsis. The culture was positive in $26(59 \%)$ with trend of E-coli in $9(20 \%)$ and Group-A streptococcus (GAS)in 5(11\%).

Among 396 patients with sepsis the adherence to survival bundle of P-sepsis depicted in TableIII. Overall, the culture was sent in half of the patients. Biomarkers like C-reactive protein was done in $25(6.3 \%)$ while serum lactate was measured in $19(5 \%)$ out of which 12 patients had the level $\geq 4 \mathrm{mmol} / \mathrm{L}$. Antibiotics were administered to $90(23 \%)$ within one hour of arrival. Sepsis with organ dysfunction was found in 44 patients, all patients were attended by consultant within three hours, among them $12(27 \%)$ had serum lactate more than $\geq 4 \mathrm{mmol} / \mathrm{L}$. Central venous pressure monitoring with fluid resuscitation was done as per protocol of survival bundle given to all $12(100 \%)$ patients with high serum lactate, while vasopressin was needed in half of these patients $6(50 \%)$.

Management of sepsis with trends to antibiotics and further intervention required to control sepsis is shown in Table-IV. Amid 44 patients of severe sepsis $29(66 \%)$ were admitted to special care, while $15(34 \%)$ required intensive care admissions. Our 7(16\%) patients expired. All of them had multi-organ failure.

Table-III: Compliance of Survival Bundle in p-sepsis.

\begin{tabular}{lc}
\hline & Total N 396(\%) \\
\hline Obtain cultures & $191(48.1)$ \\
Blood & $145(36.6)$ \\
Urine & $154(38.9)$ \\
High Vaginal swab & $97(24.5)$ \\
Wound swab & $10(2.5)$ \\
Fluid tap & $8(2.0)$ \\
Measure serum lactate/CRP & $44(11.1 \%)$ \\
Administer Antibiotics (one hour) & $90(22.7)$ \\
Severe Sepsis & Total N 44(\%) \\
Consultant arrival in 3 hours & $49(113.36)$ \\
CVO2 & $37(84.1)$ \\
Serum lactate $\geq 4$ & $12(27.2)$ \\
Fluid resuscitation & $12 / 12(100)^{*}$ \\
CVP & $12(100)^{*}$ \\
Vasopressin & $6(50)^{*}$ \\
\hline
\end{tabular}

*Denominator is 12 who had S/lactate $\geq 4 \mathrm{mmol} / \mathrm{L}$. 
Table-IV: Medical and surgical management of P-sepsis.

\begin{tabular}{lc}
\hline Antibiotics & Total N 396(\%) \\
\hline Cephalosporin and & $275(69.4)$ \\
metronidazole & $101(25.5)$ \\
Co-amoxicillin & $3(8)$ \\
clindamycin & $16(4)$ \\
Imepenum & $37(9.3)$ \\
Piperacillin & $12(3.0)$ \\
Vancomycin & $1(0.3)$ \\
Tecoplasmin & $2(0.5)$ \\
polymyxin & $N 44($ Denominator $)$ \\
Surgical management & \\
Source Control (severe sepsis) & $2(4.54)$ \\
Evacuation of uterus & $10(22.72)$ \\
TOP/LSCS & $5(11.36)$ \\
Laparotomy & $2(4.54)$ \\
Hystrectomy & $5(11.36)$ \\
Resuturing & $5(11.36)$ \\
Ultrasound guided aspiration & \\
\hline
\end{tabular}

${ }^{*} \mathrm{~N} 44$ (Denominator).

\section{DISCUSSION}

This retrospective record review in five years showed the 396 patients had P-sepsis, among them $44(11.1 \%)$ patients had severe sepsis with organ dysfunction. The studies in Pakistan showed the rate between 3.9-6.3\%, ${ }^{11,12}$ with maternal mortality related to P-Sepsis was between 13-20\%. ${ }^{13-15}$ The available data in Pakistan is limited mainly hospital based data, the criteria of sepsis in also not clear. ${ }^{16}$

This study showed that anemia was found in $63 \%$ of patients with sepsis, while other studies conducted in a public hospital showed, almost all suffering from anemia and one third of women hemoglobin was less $5 \mathrm{~g} / \mathrm{dl}$ with sepsis. ${ }^{11,12}$ Our data set, catered only to patients with sepsis and showed that $62 \%$ had delivered by C-section, hence unable to check the rate of $\mathrm{C}$-section in non-sepsis patients. An American study also exhibit the higher rate of C-section in sepsis patient with odd ratio of $1.58 .{ }^{17}$

Our study depict E-coli is the commonest organism followed by GAS. This has been further endorsed by a study in Zimbabwe, however confidential enquiries on maternal mortality UK suffice Group A streptococcus as the major culprit of p-sepsis related mortality. ${ }^{9,18}$
The compliance of Sepsis survival bundle was variable. The cultures were obtained in half of the patients with sepsis. Similarly, other components were also accomplished in severe sepsis, but not in all patients with sepsis. Multi organ failure leads to most of the death $9(78 \%)$ in our study, this was further endorsed by a local study, showed that in majority of maternal mortality is associated with Multi organ failure. ${ }^{19}$ Therefore, strict compliance to sepsis survival bundle could improve maternal morbidity and mortality. World Health Organization Global Sepsis Study (GLOSS), shows the death rate due to sepsis in low middle income(LMIC) and low income (LIC) countries range from $7-14.8 \%$ respectively, this far more then upper middle income countries(UMIC) i.e. $1.1 \% .{ }^{20} \mathrm{It}$ is a strong recommendation to take culture and start antibiotics within one hour of arrival. Therefore, broad spectrum antibiotics usually starts within one hour after collecting relevant cultures. ${ }^{7}$

The most common antibiotics used in this study was the combination of cephalosporin and metronidazole followed by Co-amoxiclav. However, this was the choice up to 2005, but due to lack of coverage against Methicillin-resistant staphylococcus aureus (MRSA), Extended Spectrum BetaLactamase (ESBL) and pseudomonas along with increased risk of C.difficile infection. Piperacillin/ tazobactam or carbapenem with clindamycin is the first choice, ${ }^{10,21}$ On the other hand these empirical antibiotics could develop multi drug resistance. ${ }^{22}$ Molecular technology has been evolving to receive culture results in < one hour. ${ }^{23}$ It has been proven from the research conducted in US health system that resuscitation survival bundle is directly correlated with the duration of compliance, by reduction in mortality by $7 \%$ in consequent quarters. Those hospitals who used this for more than three years showed reduced mortality by $36 \% .^{24}$ If the time limits to three hours, further improved the survival in cost effective manner. ${ }^{25}$ An update in 2018 by Levy suggests, more aggressive management by one hour. ${ }^{26}$ However adopting to one hour bundle is a concern, leads to more pressure on emergency department with over treatment of patients among sepsis without shock. ${ }^{22}$ Procalcitonin and C-Reactive proteins are non-specific markers. The use of broad-spectrum antibiotics empirically leads to cross resistance. The availability of specific devices that identify the causative organism and sensitivity to antibiotics in one hour is warranted. ${ }^{23}$

LMIC countries like Pakistan, being an underresourced and low resilience health system with 
inadequate staffing, low funds, lack of expertise, substandard care, absence of national guidelines and weak referral system has negative impact on the management of sepsis. Similarly, inadequate surgical and critical care capacity, misuse of antibiotics and increasing Anti-microbial resistance further worsen the situation. ${ }^{20}$ The other factors that increases the risk of sepsis are illiteracy, poverty, poor sanitation, delivery at home, inadequate transport facilities, limited resources and unequipped health facilities with limited staffing and suboptimal care.

Limitations of the study: It is retrospective record review with single hospital-based data. This is one of the private university hospitals mainly catered one socioeconomic class, so the incidence was found lower than the government hospital.

\section{CONCLUSION}

Anemia and operative deliveries are the major risk factors in sepsis. There was moderate adherence of modified surviving sepsis campaign resuscitation bundles. Further improvement in compliance warranted.

\section{Grant Support \& financial disclosure: None.}

\section{REFERENCES}

1. Trends in maternal mortality: 1990-2015: Estimates from WHO, UNICEF, UNFPA, World Bank Group \& the United Nations Population Division: Executive summary. World Health Organization; 2015.

2. Boldosser-Boesch A, Brun M, Carvajal L, Chou D, de Bernis L, Fogg K, et al. Setting maternal mortality targets for the SDGs. Lancet. 2017;389(10070):696-697. doi: 10.1016/S0140-6736(17)30337-9

3. Say L, Chou D, Gemmill A, Tunçalp Ö, Moller A-B, Daniels J, et al. Global causes of maternal death: a WHO systematic analysis. Lancet Global Health. 2014;2(6):e323-e333. doi: 10.1016/S2214-109X(14)70227-X.

4. Khan KS, Wojdyla D, Say L, Gülmezoglu AM, VanLook PF. WHO analysis of causes of maternal death: A systematic review. Lancet. 2006;367(9516):1066-1074. doi: 10.1016/S01406736(06)68397-9

5. Singer M, Deutschman CS, Seymour CW, Shankar-Hari M, Annane D, Bauer $\mathrm{M}$, et al. The third international consensus definitions for sepsis and septic shock (Sepsis-3). JAMA. 2016;315(8):801-810. doi: 10.1001/jama.2016.0287

6. Maharaj D. Puerperal pyrexia: A review. Part I. Obstet Gynecol Surv. 2007;62(6):393-399. doi: 10.1097/01. ogx.0000265998.40912.5e

7. Dellinger RP, Levy MM, Rhodes A, Annane D, Gerlach H, Opal $\mathrm{SM}$, et al. Surviving Sepsis Campaign: International guidelines for management of severe sepsis and septic shock, 2012. Crit Care Med. 2013;39(2):165-228. doi: 10.1007/s00134-012-2769-8

8. Dellinger RP, Levy MM, Carlet JM, Bion J, Parker MM, Jaeschke $\mathrm{R}$, et al. Surviving Sepsis Campaign: International guidelines for management of severe sepsis and septic shock: 2008. Intensive care medicine. 2008;34(1):17-60. doi: 10.1007/s00134-007-0934-2

9. Majangara R, Gidiri MF, Chirenje ZM. Microbiology and clinical outcomes of puerperal sepsis: A prospective cohort study. J Obstet Gynaecol. 2018;38(5):635-641. doi: 10.1080/01443615.2017.1399112.
10. Morgan M, Hughes R, Kinsella S. Bacterial sepsis following pregnancy. RCOG Green-top Guideline [Internet]. 2012.

11. Khaskheli MN, Baloch S, Sheeba A. Risk factors and complications of puerperal sepsis at a tertiary healthcare centre. Pak J Med Sci. 2013;29(4):972-976. doi: 10.12669/ pjms.294.3389

12. Madhudas C, Khurshid F, Sirichand P. Maternal morbidity and mortality associated with puerperal sepsis. JLUMHS. 2011;10(03):121

13. Fikree FF, Midhet F, Sadruddin S, Berendes HW. Maternal mortality in different Pakistani sites: ratios, clinical causes and determinants. Acta Obstet Gynecol Scand. 1997;76(7):637-645. doi: 10.3109/00016349709024603

14. Bano N, Chaudhri R, Yasmeen L, Shafi F, Ejaz L. A study of maternal mortality in 8 principal hospitals in Pakistan in 2009. Int J Gynecol. 2011;114(3):255-259. doi: 10.1016/j.ijgo.2011.03.018

15. Qureshi R, Irfan Ahmed S, Raza A, Khurshid A, Chishti U. Obstetric patients in intensive care unit: Perspective from a teaching hospital in Pakistan. JRSM Open. 2016;7(11):1-8. doi: 10.1177/2054270416663569\#

16. Organization WH. Global report on the epidemiology and burden of sepsis: current evidence, identifying gaps and future directions. 2020.

17. Al-Ostad G, Kezouh A, Spence AR, Abenhaim HA. Incidence and risk factors of sepsis mortality in labor, delivery and after birth: Population-based study in the USA. J Obstet Gynaecol Res. 2015;41(8):1201-1206. doi: 10.1111/jog.12710

18. Saving Lives, Improving Mothers' Care: Surveillance of maternal deaths in the UK 2012-14 and lessons learned to inform maternity care from the UK and Ireland Confidential Enquiries into Maternal Deaths and Morbidity 2009-14 [Internet]. 2016.

19. Bibi S, Memon A, Sheikh J, Qureshi A. Severe acute maternal morbidity and intensive care in a public sector university hospital of Pakistan. J Ayub Med College Abbottabad. 2008;20(1):109-112.

20. Knight M, Brizuela V, Abalos E, Bonet M. Frequency and management of maternal infection in health facilities in 52 countries: Results of the WHO Global Maternal Sepsis Study (GLOSS), a one-week inception cohort. Lancet Glob Health. 2020. doi: 10.1016/S2214-109X(20)30109-1

21. Bowyer L. The confidential enquiry into maternal and Child health (CEMACH). Saving mothers' lives: reviewing maternal deaths to make motherhood safer 2003-2005. The seventh report of the confidential enquiries into maternal deaths in the UK. SAGE Publications Sage UK: London, England; 2008.

22. Labib A. Sepsis Care Pathway 2019. Qatar Med J. 2019;2019(2):4 doi: 10.5339/qmj.2019.qccc.4

23. Singer M. Biomarkers for sepsis-past, present \& future. Qatar Med J. 2019; 2019(2):8. doi: 10.5339/qmj.2019.qccc.8

24. Levy MM, Rhodes A, Phillips GS, Townsend SR, Schorr CA, Beale $\mathrm{R}$, et al. Surviving Sepsis Campaign: association between performance metrics and outcomes in a 7.5-year study. Critical care medicine. 2015;43(1):3-12. doi: 10.1097/ccm.0000000000000723

25. Leisman DE, Doerfler ME, Ward MF, Masick KD, Wie BJ, Gribben JL, et al. Survival Benefit and Cost Savings From Compliance With a Simplified 3-Hour Sepsis Bundle in a Series of Prospective, Multisite, Observational Cohorts. Critical care medicine. 2017;45(3):395-406 doi: 10.1097/ccm.0000000000002184

26. Levy MM, Evans LE, Rhodes A. The surviving sepsis campaign bundle: 2018 update. Intensive care medicine. 2018;44(6):925928 doi: $10.1007 /$ s00134-018-5085-0

Authors' Contribution: FY conceived theidea, develop study protocol, responsible for accuracy, integrity of the data and prepared the manuscript. AM helped in acquisition of data, identify the key parameters to assess. She was also responsible for accuracy and integrity of the data. AS helped in Data acquisition \&manuscript editing. SS helped in proposal writing, statistical analysis and interpretation of results. 